\title{
A laser powder bed fusion system for in situ x-ray diffraction with high-energy synchrotron radiation
}

Cite as: Rev. Sci. Instrum. 91, 075104 (2020); https://doi.org/10.1063/1.5143766

Submitted: 07 January 2020 . Accepted: 09 June 2020 . Published Online: 02 July 2020

Eckart Uhlmann, Erwin Krohmer (D), Felix Schmeiser (D), Norbert Schell, and Walter Reimers

COLLECTIONS

This paper was selected as Featured
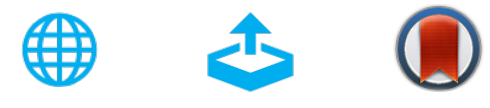

\section{ARTICLES YOU MAY BE INTERESTED IN}

Enhanced sensitivity of nanoscale subsurface imaging by photothermal excitation in atomic force microscopy

Review of Scientific Instruments 91, 063703 (2020); https://doi.org/10.1063/5.0004628

An instrument for in situ time-resolved X-ray imaging and diffraction of laser powder bed fusion additive manufacturing processes

Review of Scientific Instruments 89, 055101 (2018); https://doi.org/10.1063/1.5017236

Ion thrusters for electric propulsion: Scientific issues developing a niche technology into a game changer

Review of Scientific Instruments 91, 061101 (2020); https://doi.org/10.1063/5.0010134

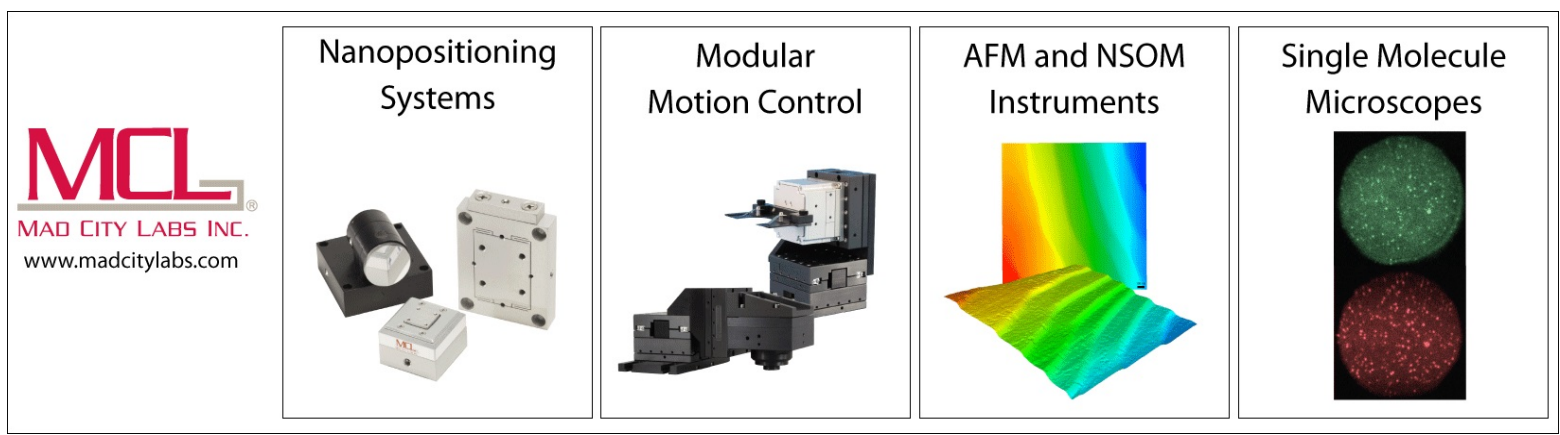




\title{
A laser powder bed fusion system for in situ $x$-ray diffraction with high-energy synchrotron radiation
}

\author{
Cite as: Rev. Sci. Instrum. 91, 075104 (2020); doi: 10.1063/1.5143766 \\ Submitted: 7 January 2020 - Accepted: 9 June 2020 • \\ Published Online: 2 July 2020
}

Eckart UhImann, ${ }^{1}$ Erwin Krohmer, ${ }^{1, a)}$ (D) Felix Schmeiser, ${ }^{2}$ (D) Norbert Schell, ${ }^{3}$ and Walter Reimers ${ }^{2}$

\begin{abstract}
AFFILIATIONS
${ }^{1}$ Institute for Machine Tools and Factory Management, Technische Universität Berlin, Pascalstraße 8-9, 10587 Berlin, Germany

${ }^{2}$ Institute for Materials Science and Technology, Metallic Materials, Technische Universität Berlin, Ernst-Reuter-Platz 1, 10587 Berlin, Germany

${ }^{3}$ Helmholtz-Zentrum Geesthacht, Max-Planck-Str. 1, 21502 Geesthacht, Germany
\end{abstract}

\begin{abstract}
a) Author to whom correspondence should be addressed: erwin.krohmer@iwf.tu-berlin.de
\end{abstract}

\begin{abstract}
In Laser Powder Bed Fusion (LPBF), the highly localized energy input by the laser leads to high-temperature gradients. Combined with the inherent cycles of re-melting and solidification of the material, they can result in high mechanical stresses. These stresses can cause distortion and cracking within the component. In situ diffraction experiments with high-energy synchrotron radiation allow an analysis of the lattice spacing during the LPBF process and provide insight into the dynamics of stress generation and texture evolution. In this work, an LPBF system for the purpose of synchrotron x-ray diffraction experiments during the manufacturing process of multi-layer components with simple geometries is described. Moreover, results from diffraction experiments at the HEMS beamline P07 at PETRA III, DESY, Hamburg, Germany, are presented. Components with a length of $l_{s}=20 \mathrm{~mm}$ and a width of $\mathrm{w}_{\mathrm{s}}=2.5 \mathrm{~mm}$ consisting of 100 layers with a layer thickness of $\Delta \mathrm{z}=50 \mu \mathrm{m}$ were produced using the nickel-base alloy Inconel 625 as the powder material. Diffraction experiments were carried out in situ at sampling rates of $\mathrm{f}=10 \mathrm{~Hz}$ with a synchrotron radiation beam size of $750 \times 70 \mu \mathrm{m}^{2}$. The presented experimental setup allows for the observation of arbitrary measuring positions in the sample in the transmission mode while gathering full diffraction rings. Thus, new possibilities for the observation of the dynamic evolution of strains, stresses, and textures during the LPBF process are provided.
\end{abstract}

Published under license by AIP Publishing. https://doi.org/10.1063/1.5143766

\section{INTRODUCTION}

The market of additive manufacturing systems for metal components has been growing rapidly in recent years ${ }^{1}$ with a focus on powder-bed based manufacturing technologies, such as Laser Powder Bed Fusion (LPBF), which is also referred to as Laser Beam Melting (LBM) or Selective Laser Melting (SLM). LPBF offers the possibility to design and manufacture components with complex geometries while maintaining good mechanical properties. However, the process stability still needs improvement as the limited reproducibility inhibits the breakthrough of LPBF in fracture-critical applications. ${ }^{2}$ Current challenges include crack-inducing defects or residual stresses ${ }^{4}$ and require non-destructive testing methods and material qualification processes. ${ }^{5}$ In order to avoid the formation of defects and to control the residual stress states, further knowledge considering their origins and a profound understanding of the physical phenomena in melt-pool creation, solidification, and microstructural evolution is needed. At this point, simulation models help us to understand and to predict residual stress, ${ }^{6}$ defect formation, and microstructure. ${ }^{8}$ However, those models require experimental validation. Due to the small timescales of melting and solidification processes during LPBF, highly dynamic monitoring procedures are required to collect experimental data with sufficient temporal resolution. Thermal metrology based melt-pool monitoring systems have been widely addressed in research, ${ }^{9}$ and some are state of the art in commercial LPBF systems. They provide important insights into the dynamics of the process. Yet, those are surface related measurement techniques with limited suitability to gather information about bulk properties. Hence, new in situ measurement methods are necessary, especially in the challenging case of 
the investigation of residual stress evolution and varying metallic phase structure. ${ }^{10}$ With the development of the third-generation synchrotron light sources, in situ diffraction experiments during time-critical manufacturing processes became possible. ${ }^{11}$ Together with $\mathrm{x}$-ray imaging data, $\mathrm{x}$-ray diffraction with high-energy synchrotron radiation offers bulk sensitive insights into the melting and solidification process in LPBF.

Only recently researchers presented their work on in situ $\mathrm{X}$ ray diffraction and imaging in LPBF. Uhlmann et al. ${ }^{12}$ discussed the complex implementation of such an experimental setup and the requirements that the LPBF system should meet to simulate an industrial system. Bidare et al. ${ }^{13,14}$ presented a compact system with access for $\mathrm{x}$ rays and suitable for several in situ imaging techniques such as high-speed schlieren imaging. The first results from in situ $\mathrm{x}$ ray diffraction and in situ $\mathrm{x}$-ray imaging experiments with a system that mimics LPBF conditions were reported by Zhao et al. ${ }^{15}$ They observed dynamic melt pool evolution, keyhole pore formation, and the motion of ejected particles in the process of melting and solidification of Ti6Al4V powder with a frame rate of $f=50 \mathrm{kHz}$. In addition, they showed the possibility to investigate phase transformations by means of x-ray diffraction. Subsequently, the described LPBF system was upgraded and used in several further investigations so that high-speed $\mathrm{x}$-ray imaging with a frame rate of up to $6.5 \mathrm{MHz}$ proved possible to give profound insight into the transient dynamics of the LPBF process. ${ }^{16}$ Guo et al. ${ }^{17}$ continued the research on powder spattering behavior and evaluated the dynamics of powder spattering as a function of time, ambient pressure, and location. Additional research on powder motion was conducted by Escano et al. ${ }^{18,19}$ who designed a device to examine the powder deposition by a spreading wiper by means of high-speed $\mathrm{x}$-ray imaging. Another setup that allows the investigation of phase transformations and lattice expansion during cyclic heating and cooling using micro-x-ray diffraction was introduced by Kenel et al. ${ }^{20}$ The setup simulates the thermal behavior of a multi-layer LPBF process but under considerably different conditions than in a common industrial system as no powder material is involved. Nevertheless, the advantage of the presented setup is the in situ gathering of surface temperature information. ${ }^{21}$ Leung et al. ${ }^{22}$ used a custom-built system called LAMPR, short for Laser Additive Manufacturing Process Replicator, for in situ $\mathrm{x}$-ray imaging experiments. Research with the LAMPR includes time-resolved melt track, spatter, and defect formation of different materials. ${ }^{22-24}$ Calta et al. ${ }^{25}$ developed a system for the purpose of in situ $\mathrm{x}$-ray imaging and diffraction of single tracks. They reported experiments showing pore formation via in situ $\mathrm{x}$-ray imaging and $\beta$-Ti- $\alpha$-Ti phase transitions upon cooling in Ti6Al4V via $\mathrm{x}$-ray diffraction. In further studies, utilizing the mentioned experimental systems, melting and solidification dynamics in single tracks of Aluminum 6061 and AISI 4140 steel $^{26,27}$ and pore formation and spatter dynamics in the laser-metal-interaction with solid and powdery Ti6Al4V ${ }^{28-31}$ were investigated. Finally, Hocine et al. ${ }^{32}$ presented an advanced experimental instrumentation for in situ diffraction experiments. Their instrumentation adopts a hopper-based powder feeding system and a particle filter system for the inert gas circulation and is therefore applicable for multi-layer experiments. They analyzed the influence of laser processing parameters and scanning strategies on the phase transformation and microstructural evolution in several Ti6Al4V samples in the reflection mode.
In this paper, an experimental system for in situ x-ray diffraction with high-energy synchrotron radiation is presented, which is designed for the investigation of the evolution of textures and residual stresses during the build process of three-dimensional parts. With the presented setup, it is possible to produce multi-layer parts with conditions mostly similar to the industrial LPBF process. This system's key advantages are the possibility to gather full diffraction rings to improve measurement accuracy, the free choice of the measuring position in the sample, and the feasibility of various measurement modes that deliver a range of spatially and temporally resolved data. Further experimental results using the here described experimental setup have been discussed in depth by Schmeiser et $a l^{3.3}$

\section{SYSTEM DESCRIPTION AND SPECIFICATIONS}

\section{A. Instrument design}

The experimental instrumentation consists of an LPBF system positioned in the x-ray beam path of a synchrotron light source such that a desired gauge volume in the sample is irradiated and the diffracted $x$ rays are detected by a $2 \mathrm{D}$ detector, e.g., scintillatorbased. The experimental approach is depicted in Fig. 1. The customized LPBF system, which allows for in situ x-ray diffraction corresponding to the described experimental geometry, is presented below. The specified system was designed to operate at the HEMS beamline P07 at PETRA III. ${ }^{34}$ Hence, any diffraction related specifications or described peripheral equipment refer to the beamline's facilities.

One of the main requirements during the design process was to reproduce the conditions of a commercial LPBF setup as precisely as possible while accounting for the required $\mathrm{x}$-ray transparency. First, attenuation of $\mathrm{x}$ rays throughout the experimental setup, besides in the sample, should be avoided in order to prevent noise in the measurement. Therefore, compared to a state of the art industrial LPBF system, the powder bed is elevated and unavoidable objects in the $\mathrm{x}$-ray path are made of material with high $\mathrm{x}$-ray transparency. Furthermore, the system features an additional linear axis, ensuring linear motion of the powder bed not only vertically but also horizontally, perpendicular to the incident synchrotron radiation beam. Since the synchrotron radiation beam and the detector are stationary, the powder bed, therefore, can be positioned relative to the measuring instrumentation without moving the whole process chamber. Thus, free variation of the measuring position is facilitated, and different measurement modes can be implemented.

The InSituLPBF system presented here is based on the modular industrial machine AconityMINI manufactured by AconiTy3D GMBH, Herzogenrath, Germany. The process chamber was designed, built, and integrated into the industrial system at the INSTITUTE FOR MACHINE TOOLS AND FACTORY MANAGEMENT Of TECHNISCHE UNIVERSITÄT BERLIN in order to meet the requirements for in situ x-ray diffraction experiments. The system consists of three modules. Control systems for media and energy supplies as well as the laser source are situated in the first module, the control cabinet, which also serves as the human-machine interface. The second module is the process chamber itself. The third module contains the components for circulation and filtration of the inert gas atmosphere in the process chamber. For in situ x-ray diffraction experiments, the process chamber is mounted on a heavy load hexapod from PHYsIK INSTRUMENTE (PI) GMBH 


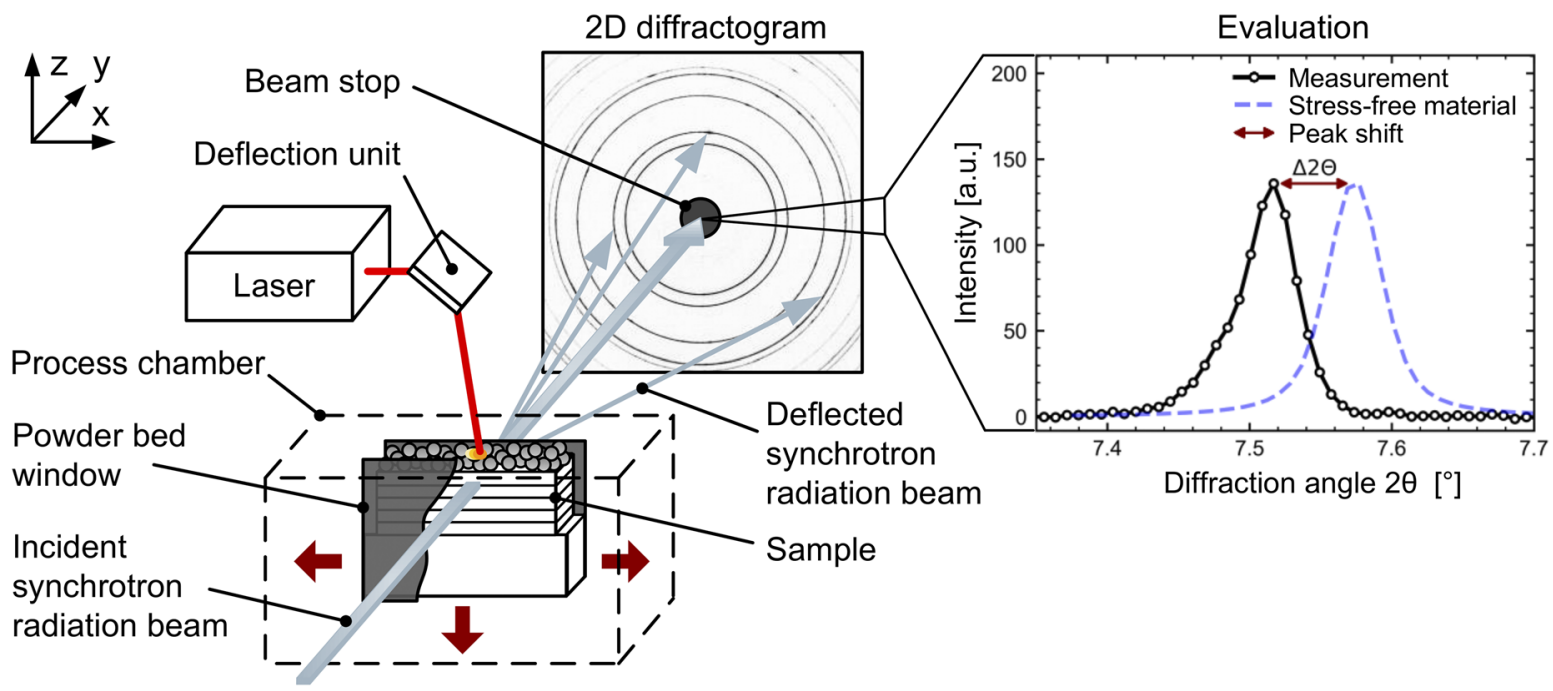

FIG. 1. Schematic of the experimental approach for $\mathrm{x}$-ray diffraction with synchrotron radiation.

\& CO. KG, Karlsruhe, Germany, which is part of the Experimental Hutch 3 at the HEMS beamline P07. The laser source is a singlemode continuous wave Ytterbium fiber laser YLR-400-AC from IPG LASER GMBH, Burbach, Germany, which emits radiation at a wavelength of $\lambda_{\mathrm{L}}=1070 \mathrm{~nm}$ with a nominal power output of $\mathrm{P}_{\mathrm{L}}=400 \mathrm{~W}$. The laser fiber is connected to the three-axis deflection unit Axialscan-30 from RaYlase, Wessling, Germany, via a collimator. The installed deflection unit features a focus compensation and scanning speeds of $\mathrm{v}_{\mathrm{L}}=3 \mathrm{~m} / \mathrm{s}$ can be realized. A cooling unit SC11 from GLEN Dimplex DeUTSCHLAND GMBH, Kulmbach, Germany, provides the scan head with coolant for the galvo mirrors. The laser beam has a Gaussian shape with an adjustable focus diameter between $\mathrm{d}_{\mathrm{L}} \approx 60 \mu \mathrm{m}$ and $500 \mu \mathrm{m}\left(1 / \mathrm{e}^{2}\right)$ and is deflected onto a powder bed with a length of $\mathrm{l}_{\mathrm{b}}=70 \mathrm{~mm}$ and a width of $\mathrm{w}_{\mathrm{b}}=3 \mathrm{~mm}$. The optical working distance of the setup is $455 \mathrm{~mm}$. The powder bed is enclosed by two glassy carbon plates, HTw HOCHTEMPERATUR-WERKSTOFFE GMBH, Thierhaupten, Germany, each with a thickness of $1 \mathrm{~mm}$ in the x-ray transmission direction. The samples are built on a replaceable substrate plate, which is sandwiched between the glassy carbon plates. The material of the substrate is selected according to the powder material. The sample holder together with the glassy carbon mount is depicted in Fig. 2. Here, substrate plates with the dimensions of $70 \times 10 \times 3 \mathrm{~mm}^{3}$ are mounted in the groove of the stainless steel build plate. The surrounding material is PAMITHERM 41140, Von ROLl DEUTSCHLAND gMBH, Augsburg, Germany, a high-temperature resistant silicone phlogopite mica laminate to thermally insulate the carriage from the installed heating ceramic, BACH RC GMBH, Seefeld, Germany, which is in contact with the bottom side of the build plate. The substrate plate can be heated up to $300{ }^{\circ} \mathrm{C}$. If the desired gauge volume is in a lateral distance of more than $10 \mathrm{~mm}$ to either side of the glassy carbon mount, the sample holder allows for Bragg angles $2 \theta \leq 14^{\circ}$ without shadowing of the diffracted $\mathrm{x}$ rays. The detailed view in Fig. 2(a) shows a schematic illustration of the powder bed, which is confined between the two glassy carbon plates and the substrate beneath during an experiment. The sample holder is mounted on a rigid guide rail, which is actuated in the $\mathrm{z}$-axis direction by an EMC electromechanical cylinder, вOSCH REXROTH AG, Lohr am Main, Germany. Additionally, the sample holder is actuated in the $\mathrm{X}$-axis direction by a C-shaped driver, which is mounted on a CKK-110 linear axis, Bosch ReXroth AG, Lohr am Main, Germany. The motion arrangement comprises a sliding contact between the C-shaped driver and the sample holder such that the movements of the two linear axes are decoupled. As a result, the C-shaped driver is fixed in the $\mathrm{z}$-direction. This principle assures a constant working plane, as the glassy carbon plates are mounted on the driver.

When the sample holder moves in the positive z-direction, the glassy carbon plates stay in position, leading to a void between the substrate plate and the edges of the glassy carbon. The fully automatic recoating mechanism fills the void with powder and levels it. The operating principle is demonstrated in Fig. 3. The powder recoating mechanism is based on a funnel, which is actuated in the $\mathrm{x}$ axis direction by another CKK-110 linear motion axis. For recoating, the sample holder is moved to the recoating position [see Fig. 3(a)]. Then, the funnel is moved over the sample holder up to its turning point. During motion, the powder flows into the void due to gravity alone [see Fig. 3(b)]. On its way back, the elastomeric lip attached to the funnel levels the powder layer, as depicted in Fig. 3(c), and scrapes the excess powder to the sides of the sample holder. The gap between the funnel and glassy carbon plates is usually set to $\sim 100 \mu \mathrm{m}$ but can be adjusted manually using the funnel height adjustment and precision sheet-metal. Returned to its parking position, the funnel orifice is sealed by the thick powder layer beneath. The funnel shape was tested and optimized for titanium and nickelbase alloys with a particle size distribution of $20 \mu \mathrm{m}-63 \mu \mathrm{m}$ and a spherical particle shape. Note that, e.g., finer powder or different materials could lead to bridging in the orifice due to cohesiveness of the powder. With the use of small vibration motors, which were installed on the funnel and are not depicted in Fig. 3, the flowability of the powder was improved and formerly clogging powders 

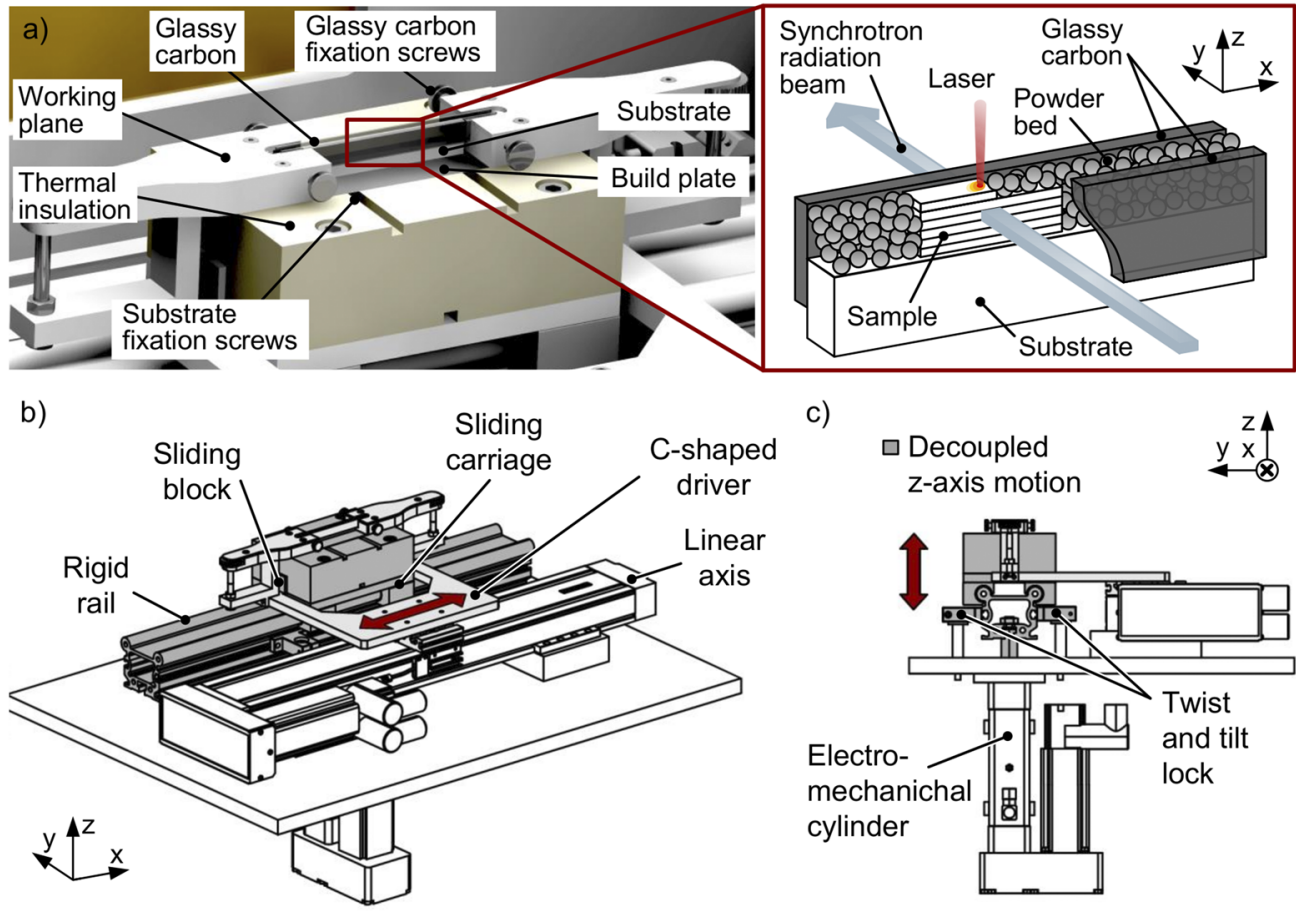

FIG. 2. Sample holder: (a) rendering of the sample holder and schematic of the sample during the experiment, (b) linear axis for $x$-axis motion, and (c) linear axis for $z$-axis motion.

could be processed as well, e.g., TNB-V5 powder. Using an extension, the powder capacity of the funnel can be increased as desired. With a layer thickness of $\Delta \mathrm{z}=50 \mu \mathrm{m}$, parts consisting of 120 layers or approximately a height of $h_{s}=6 \mathrm{~mm}$ were produced so far utilizing such a powder capacity extension. The motion limits of the $\mathrm{z}$-axis allow for maximum part heights of $\mathrm{h}_{\mathrm{s}}=10 \mathrm{~mm}$ or 200 layers when using a layer thickness of $\Delta \mathrm{z}=50 \mu \mathrm{m}$.

For laser processing, the process chamber has to be sealed and purged with an inert gas, usually argon. For this purpose, the process chamber has an inlet and outlet port for inert gas flooding. The excess pressure during flooding and laser melting process is constantly controlled by the proportional pressure regulator VVPM, FESTO AG \& CO. KG, Esslingen, Germany, while the gas flow is monitored by the installed flow sensor SFAB, FeSTO AG \& CO. KG, Esslingen, Germany. Both components are part of the basic AconityMINI system. The ambient conditions inside the process chamber are monitored by means of an oxygen sensor and a thermocouple. Oxygen values and excess pressure and inert gas flow data are logged on the computer besides other data such as axis positions and laser status. Two further ports in the process chamber walls serve as inlet and outlet
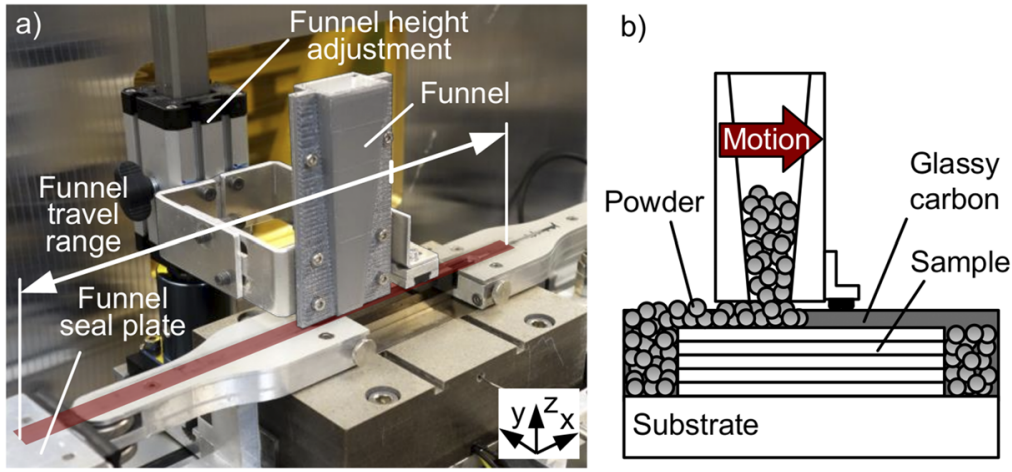

c)

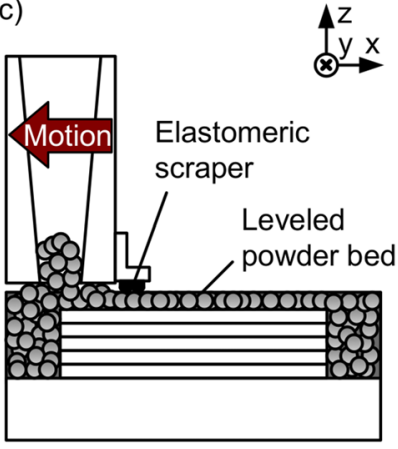

FIG. 3. Functionality of the powder recoating mechanism: (a) positioning, (b) filling with fresh powder, and (c) leveling the powder bed. 
for the continuous smoke gas extraction and filtration. The recirculation and filtration of the inert gas atmosphere ensures that the process chamber is mostly free of welding fumes and particles that might interfere with either laser or $\mathrm{x}$-ray beam and cause disturbances during the measurement. The power of the circulation pump can be regulated to adjust the flow speed.

The inlet and outlet windows of the process chamber for the incident and diffracted $\mathrm{x}$ rays are made of Kapton foils, DUPONT, INC., Wilmington, USA, with a thickness of $50 \mu \mathrm{m}$. As diffuse laser radiation might pass through the Kapton foil windows, laser safety measures need to be taken before emitting radiation. Besides the desired metal sample, the synchrotron radiation beam passes through ambient air, Kapton foils, argon, and glassy carbon with distinguished $\mathrm{x}$-ray transparency and negligible noise.

\section{B. Measurement modes}

For the synchrotron $\mathrm{x}$-ray diffraction experiments described here, a two-dimensional area detector is used. In combination with the use of monochromatic synchrotron radiation, the diffraction images deliver no information about lattice plane spacings in the transmission direction, which is therefore neglected in subsequent explanations. Up to this point, three different measurement modes are possible to conduct with the InSituLPBF system at PETRA III (see Fig. 4). In measurement mode 1 (MM1), the gauge volume (GV) is in a defined distance to the topmost layer. Relative to the sample, the vertical position of the gauge volume changes layer by layer, whereas the lateral position is fixed throughout the buildup of one sample.

In measurement mode 2 (MM2), the gauge volume corresponds to a fixed volume in the sample, which can be monitored during the melting and solidification of all the above layers. For this mode, the $\mathrm{z}$-axis translation of the beamline hexapod compensates the $\mathrm{z}$-axis translation of the powder bed and therefore ensures a fixed measurement height.

In the third measurement mode (MM3), the gauge volume is in a defined lateral and vertical distance to the laser spot. This measurement mode is limited to samples with scan tracks longitudinal to the incident synchrotron radiation beam. After the laser scans a longitudinal track, the sample holder is moved translationally according to the hatch distance $h$, before the laser scans again. As a result, the process time increases significantly compared to the other measurement modes.

\section{Data preparation}

The scan path of the laser is provided in a Common Layer Interface (CLI) file, where the scan vectors are listed in sequence for each layer separately. They are defined by their starting and ending coordinates in the working plane. An algorithm to generate CLI-files of simple cuboid samples was implemented in MATLAB 2017b, The Mathworks, INC., Natick, USA. Its input parameters are length $l_{s}$, width $\mathrm{w}_{\mathrm{s}}$, height $\mathrm{h}_{\mathrm{s}}$, layer thickness $\Delta \mathrm{z}$, hatch distance $\mathrm{h}$, and orientation of the scan vectors of the desired sample. In this study, the laser scan tracks were oriented either parallel or perpendicular to the incident synchrotron $\mathrm{x}$-radiation beam such that either transverse or longitudinal strains relative to the scan track can be evaluated in situ. Unlike in commercial data preparation for LPBF parts, there is only

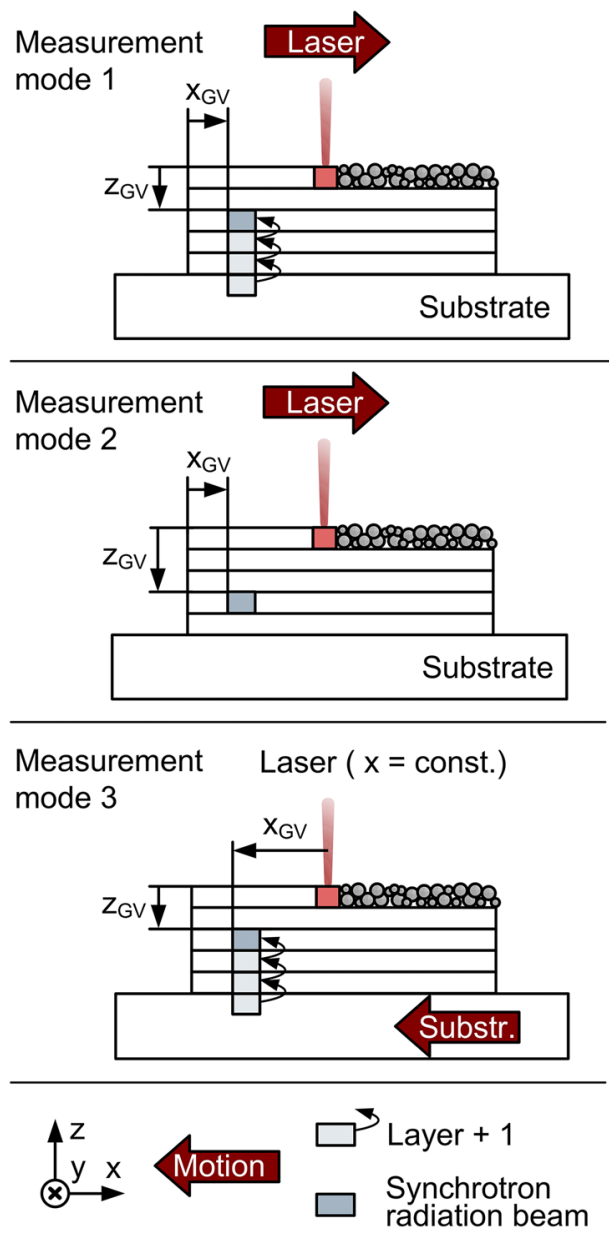

FIG. 4. Feasible measurement modes with the InSituLPBF system at PETRA III.

one type of scan vector defined. However, using certain commercial Computer Aided Manufacturing (CAM) software with the capability to generate CLI files, the definition and production of more complex samples with several vector types would be possible, as long as the powder bed size of the experimental system is not exceeded. For the sake of ease of interpretation, only unidirectional, parallel scanning patterns were utilized in the experiments conducted so far.

\section{EXPERIMENTAL PROCEDURE}

\section{A. Sample and system preparation}

In order to produce a new sample, a substrate plate has to be inserted into the groove of the build plate and fixed via two headless screws. After placing the two glassy carbon plates into the slots of the carriage and fixing them, the sample holder is ready for initial powder coating. Although the recoating procedure runs fully automatic during the LPBF process, the initial layer has to be applied 
semi-automatically as thinly as possible to guarantee sufficient bonding of the first layer with the substrate. Before each experiment, the chamber is sealed and purged with the argon gas. With the installed oxygen sensor, the oxygen level is monitored throughout the entire process. As soon as the oxygen content reaches a level below $2000 \mathrm{ppm}$, the circulation pump can be turned on to remove welding fumes from the laser beam path during the manufacturing process. In order to set the measuring position, the left, right, and upper edge of the first layer are determined by incrementally collecting diffraction patterns after laser scanning of the first layer. This can be necessary as scan track widths and therefore sample geometries may vary with changing laser scanning parameters. The measuring position can then be set accordingly in relation to the first layer's edges by adjusting (a) the position of the process chamber to set the vertical distance of the gauge volume to the top layer and (b) the position of the sample holder in the process chamber to set the lateral measuring position. Eventually, the in situ diffraction experiment can be started.

\section{B. X-ray diffraction experiments}

The x-ray diffraction experiments were conducted at the P07 HEMS beamline at PETRA III at Deutsches Elektronen-Synchrotron (DESY), ${ }^{34}$ where the process chamber module was mounted on the heavy load 6-axis positioning system (see Fig. 5). The material used in the experiments was Inconel 625 powder from M4P MATERIAL SOLUTIONS GMBH, Magdeburg, Germany, with a particle size distribution of $20 \mu \mathrm{m}-63 \mu \mathrm{m}$. The layer thickness was set to $\Delta \mathrm{z}=50 \mu \mathrm{m}$. Samples consisting of 100 layers, resulting in samples with a height of $\sim \mathrm{h}_{\mathrm{s}}=5 \mathrm{~mm}$, a length of $\mathrm{l}_{\mathrm{s}}=20 \mathrm{~mm}$, and a thickness of $\mathrm{w}_{\mathrm{s}}=2.5 \mathrm{~mm}$ in the transmission direction, were produced during the in situ measurements. To account for the thermal boundary conditions in an industrial scale LPBF machine, a powder-filled gap of $250 \mu \mathrm{m}$ was left on either side of the sample and the powder-bed limiting glassy carbon plates. Regarding the synchrotron radiation beam, the irradiated powder was factored in during the subsequent data analysis by excluding data points showing less than $40 \%$ of the respective maximum peak intensity. In the described experiments, the powder contributes to about $\mathrm{w}_{\mathrm{p}, \text { irr }}=10 \mathrm{wt}$. $\%$ of the irradiated mass, calculated using Eq. (1) with respect to the gauge volume and the irradiated

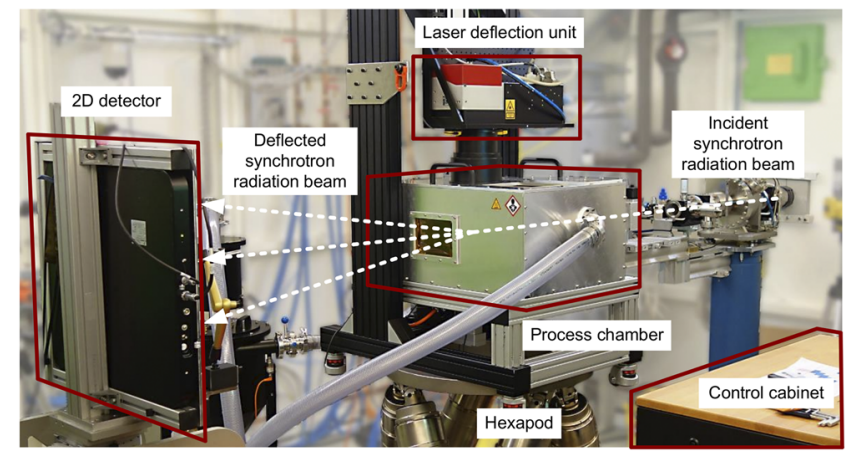

FIG. 5. Experimental setup at HEMS beamline P07 at PETRA III, DESY, Hamburg, Germany. volumes of the solid $V_{s, \text { irr }}$ and the powder $V_{p}$,irr. The irradiated volumes $V_{s, \text { irr }}$ and $V_{p \text {,irr }}$ are calculated according to the chosen gauge volume of $750 \times 70 \mu \mathrm{m}^{2}$, the thickness of the sample $\mathrm{w}_{\mathrm{s}}$, and the combined thickness of the powder gaps. They equal $\mathrm{V}_{\mathrm{s}, \text { irr }}=$ $0.131 \mathrm{~mm}^{3}$ and $\mathrm{V}_{\mathrm{p} \text {,irr }}=0.026 \mathrm{~mm}^{3}$. Including an error estimation of $3 \%$ for possibly varying values for the solidified material density $\rho_{\mathrm{s}}=8.5 \mathrm{~g} / \mathrm{cm}^{3}$ and the bulk density of the powder $\rho_{\mathrm{p}}=4.5 \mathrm{~g} / \mathrm{cm}^{3}$, we derive a maximum fraction of $\mathrm{w}_{\mathrm{p} \text {,irr }}=13 \mathrm{wt}$. $\%$ of the irradiated mass for the powder material,

$$
\mathrm{w}_{\mathrm{p} \text {,irr }}=\frac{\mathrm{V}_{\mathrm{p}, \text { irr }} \cdot \rho_{\mathrm{p}}}{\mathrm{V}_{\mathrm{p}, \mathrm{irr}} \cdot \rho_{\mathrm{p}}+\mathrm{V}_{\mathrm{s}, \mathrm{irr}} \cdot \rho_{\mathrm{s}}} .
$$

The samples were manufactured using a laser power of $\mathrm{P}_{\mathrm{L}}=55$ $\mathrm{W}$, a scanning velocity of $\mathrm{v}_{\mathrm{L}}=50 \mathrm{~mm} / \mathrm{s}$, a laser beam diameter of $\mathrm{d}_{\mathrm{L}} \approx 60 \mu \mathrm{m}(1 / \mathrm{e})^{2}$, and a unidirectional, longitudinal scanning pattern with respect to the synchrotron radiation beam direction.

Furthermore, MM1 was employed, so the gauge volume stayed in a constant distance to the top surface of the sample. The laser scan time $t_{L}$ indicates the time during which the laser emits radiation, i.e., exposes the current powder layer. With the given parameters, the total laser scan time $t_{\mathrm{L}, \mathrm{t}}$, which is the duration of scanning of one single layer, is $t_{\mathrm{L}, \mathrm{t}}=7.8 \mathrm{~s}$.

For the experiments, an x-ray energy of $98.02 \mathrm{keV}$ $(\lambda=0.12649 \AA)$ was used, and the beam size was set to $750 \times 70 \mu \mathrm{m}^{2}$. The beam height of $70 \mu \mathrm{m}$ ensured for sufficient spatial resolution close to the layer thickness. Combined with the chosen beam width of $750 \mu \mathrm{m}$, it was ensured that enough grains were irradiated in order to collect full diffraction rings. The diffraction patterns were collected with the Perkin-Elmer XRD1621 2D detector with an exposure time of $\mathrm{t}=0.1 \mathrm{~s}$ and a sampling rate of $\mathrm{f}=10 \mathrm{~Hz}$. Furthermore, a calibration measurement was performed using $\mathrm{a} \mathrm{LaB}_{6}$ powder sample fixated between the glassy carbon plates in the process chamber. The collection of calibration diffraction patterns using the same $\mathrm{x}$-ray diffraction exposure parameters as during the in situ experiments enabled the precise determination of the sample-to-detector distance and the detector tilt. The detector delivers $2 \mathrm{D}$ intensity distributions with a pixel resolution of $2048 \times 2048$ and a pixel size of $200 \times 200 \mu \mathrm{m}^{2}$. For polycrystalline materials such as metals, the $\mathrm{x}$-ray beam is diffracted as a number of cones onto the flat panel detector, resulting in a diffraction pattern of a set of concentric rings. The radius of the diffraction ring is proportional to the Bragg angle, which, in turn, is used to calculate the interatomic spacing following the Bragg equation,

$$
2 \mathrm{~d} \cdot \sin \theta=\mathrm{n} \cdot \lambda
$$

In case the interatomic spacing for a stress-free sample is known, deviations from the measured spacing can be used to calculate strains and stresses in the observed gauge volume.

During the in situ measurements, diffraction patterns are collected continuously by using the post-trigger function of the beamline's control software. Once the image acquisition is started, a single dark image is taken, which is subsequently subtracted from all of the diffraction patterns. During the LPBF process, the sample moves in and out of the desired gauge volume as a consequence of the recoating procedure. Image collection during that time does not generate useful data, which is why the simultaneously logged positioning 
information of the sample holder is used to select for the applicable images during post-processing.

\section{EVALUATION OF EXPERIMENTAL RESULTS}

The diffraction patterns are segmented into equal size cake pieces, which correspond to the principal directions of the sample geometry. Azimuthal angles of $\eta=0^{\circ}$ and $\eta=180^{\circ}$ equal the transverse direction (TD) with respect to the synchrotron radiation beam direction, while the build-up direction of the sample (BD) is characterized by $\eta=90^{\circ}$ and $\eta=270^{\circ}$. These cake pieces are then integrated using the software Fit $2 \mathrm{D}^{35}$ to gather $1 \mathrm{D}$ line profiles from the $2 \mathrm{D}$ data. The $1 \mathrm{D}$ line profiles are subsequently partially fitted by a Voigt function employing custom Python scripts utilizing the "Imfit" package. ${ }^{36}$ The fit function delivers a parametric description of the peaks and is used to extract the peak position, peak intensity, full width at half maximum (FWHM), and associated errors. From the peak positions and the stress-free lattice parameter $\mathrm{d}_{0}$, the lattice strain $\varepsilon$ is calculated and can be plotted over different timescales. The stress-free lattice parameter $\mathrm{d}_{0}$ was determined from stress-free cuboid samples in a separate experiment. ${ }^{33}$ For facecentered cubic materials such as Inconel 625, generally the fourth diffraction ring, corresponding to the 311 lattice plane, is analyzed as its stress state correlates well with the macroscopic stress of the material.

Due to the sample's geometry, a triaxial stress state must be assumed. Therefore, the measured strains in TD and BD are affected by the stress in the longitudinal direction (LD). The experimental geometry used here, however, does not deliver any information regarding the longitudinal direction. Nevertheless, the argument in the following paragraphs is based on the differences in the strain progressions in TD and BD. Since the contribution of LD is equal to both respective directions, the difference between $\mathrm{TD}$ and $\mathrm{BD}$ is unaffected by LD. Therefore, valuable conclusions can be drawn even without the knowledge of the full triaxial stress state.

In Fig. 6, the average strain progression during the production of cuboid samples over the course of manufacturing is shown. This figure illustrates three subplots (a)-(c), which show different measuring positions according to Fig. 6(d). For each subplot, one individual cuboid sample [Fig. 6(e)] was additively manufactured and measured layer after layer with a total number of layers of $\mathrm{n}=100$ using the parameters given in Fig. 6(f). One diagram summarizes the data from layers 20 to 100 by plotting the lattice strains $\varepsilon^{311}$ in both TD and BD over the laser scan time $t_{L}$. There are no data before or after laser emission. For the diagrams presented here, the measurements from layer 1 to 19 were omitted in order to observe stable process conditions. In LPBF, the effective layer thickness of the deposited powder layer and therefore also the solidified layer typically varies over the first few layers. ${ }^{14}$

While the colored areas show the full range of strain values for each point in time for the layers 20-100, the lines represent the corresponding median strain value. The number of measurements for each diagram is about $80 \times 78=6240$ measurements, with 80 layers consisting of 78 captures each. With respect to Fig. 4, the lateral distance of the gauge volume was $\mathrm{x}_{\mathrm{GV}}=1 \mathrm{~mm}, \mathrm{x}_{\mathrm{GV}}=10 \mathrm{~mm}$, and $\mathrm{x}_{\mathrm{GV}}=19 \mathrm{~mm}$. Thus, the peripheral measuring positions were located $1 \mathrm{~mm}$ away from the edge of the sample. In each measuring position, a clear peak in the strains is visible in both TD and $\mathrm{BD}$. At that point in time, the laser passes over the gauge volume, which is why the peak occurs first in the left gauge volume, then in the center of the sample and finally in the right edge gauge volume. The peak strains during the laser passage vary by the measuring position. For the center and right edge gauge volume, the median strain in TD during the peak is about $\varepsilon_{\mathrm{TD}}^{311}=0.8 \%$, while in the left gauge volume, it reaches only about $0.4 \%$.

This is caused by differences in the heat flow rate, which is illustrated in Fig. 7. The material in the left gauge volume is heated first during the laser scan of a layer. All of the surrounding material, i.e., powder, previously solidified layers below and the substrate plate, is cold. Therefore, heat conduction happens rapidly, leading to a lower degree of lattice expansion. For the center and right edge gauge volumes, the surrounding material, on average, has a higher temperature than for the left gauge volume since the left side of the corresponding gauge volume was already heated up, reducing the temperature gradients and, thus, slowing down the heat conduction. This type of lateral heat accumulation was also shown in simulative results by Parry et al. ${ }^{6}$ The described effects lead to higher peak temperatures in the gauge volume, resulting in higher lattice strain peaks.

While the peak itself is caused by a rise in temperature, the laser does not affect the two directions TD and BD in the same magnitude. For all of the measuring positions, the median strain values in $\mathrm{TD}$ are higher than in $\mathrm{BD}$. Since the strain values for TD and $\mathrm{BD}$ for each point in time are calculated from the same diffraction pattern, they represent grains with the same temperature. Therefore, both directions are affected by the temperature and the subsequent thermal expansion the same way, which implies that any strain disparity between the two is purely elastic. Hence, the difference between the directions has to be caused by mechanical stresses, which themselves are a result of the thermal gradient mechanism. ${ }^{38}$ When the top layer of the part is exposed to the laser, its temperature increases rapidly. The material expands, but the colder material below hinders the thermal expansion due to its lower temperature. As a consequence of the high temperature in the top layer, the material plasticizes and is compressed plastically. After being exposed by the laser, the material cools down and contracts. Caused by the plastic compression during heating, the contraction is now hindered and tensile stresses are introduced. This effect is much more pronounced in the working plane than out of plane, which results in higher tensile stresses in TD. As a result, there is a transverse contraction in BD leading to lower lattice strains. Below the plasticized zone, the material is expanded by the hotter material above, which adds another tensile effect on the gauge volume. The gauge volume with a distance of $\mathrm{z}_{\mathrm{GV}}=150 \mu \mathrm{m}$ to the top surface most likely experiences a combination of both effects.

The magnitudes of this effect are dependent on the lateral position of the gauge volume. In the center of the sample, this effect is the strongest, resulting in the highest difference between the strains in TD and BD. This holds true for both before and after the laser passes over the gauge volume. The difference before the laser passes over the gauge volume originates from the manufacturing of the previous layer, where the stresses were induced after the laser scan. Then, after the laser passage, the effect is repeated and stresses are induced again due to the process discussed above. Close to the edges, the strain difference is smaller than in the center before the laser passes over 

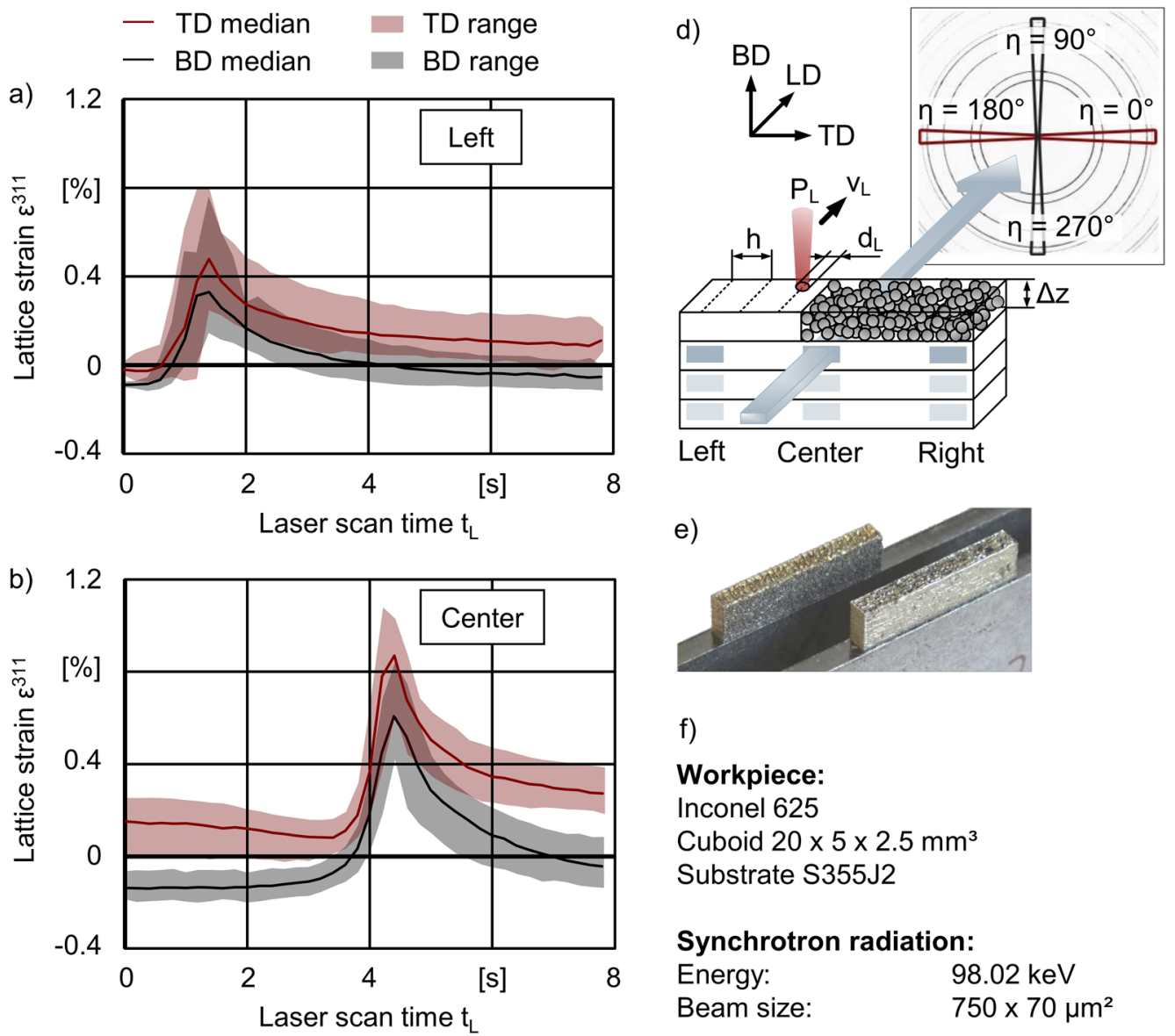

f)

\section{Workpiece:}

Inconel 625

Cuboid $20 \times 5 \times 2.5 \mathrm{~mm}^{3}$

Substrate S355J2

Synchrotron radiation:

$\begin{array}{ll}\text { Energy: } & 98.02 \mathrm{keV} \\ \text { Beam size: } & 750 \times 70 \mu \mathrm{m}^{2}\end{array}$

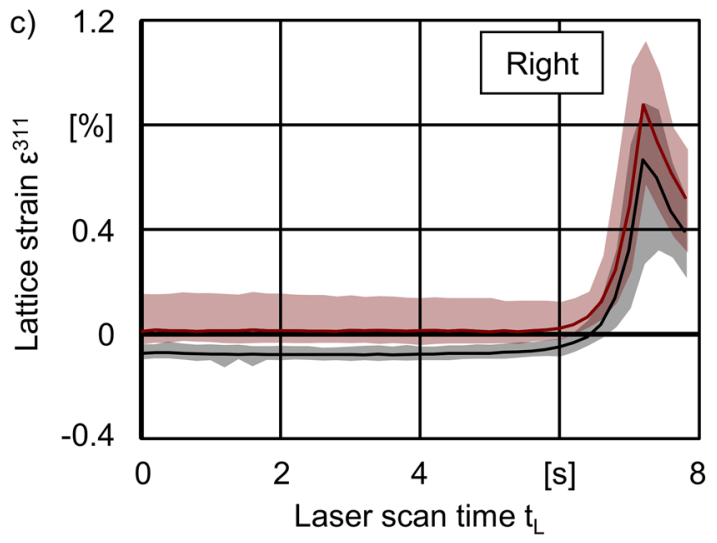

Measurement:

Mode 1

Top surface distance $\mathrm{z}_{\mathrm{GV}}=150 \mu \mathrm{m}$

Parameters:

Laser power

Scanning speed

$\mathrm{P}_{\mathrm{L}}=55 \mathrm{~W}$

Laser beam

$\mathrm{V}_{\mathrm{L}}=50 \mathrm{~mm} / \mathrm{s}$

diameter

Hatch distance

Layer thickness

$\mathrm{d}_{\mathrm{L}} \approx 60 \mu \mathrm{m}$

$\mathrm{h}=120 \mu \mathrm{m}$

Scanning pattern:

Longitudinal to synchrotron radiation beam

FIG. 6. Strain progression in cuboid samples made from Inconel 625. Gauge volumes were located (a) close to the left edge, (b) in the center, and (c) close to the right edge of the sample in three separate experiments. (d) Schematic diagram of the experiment. (e) Cuboid samples. (f) Process and measurement parameters.

the gauge volume. Due to the proximity of the edge gauge volumes to both the top surface and side faces of the sample, the free surface allows for free expansion and contraction in TD, resulting in minimal stress build-up. This is further underlined by the median strain value in $\mathrm{TD}$, which is close to $\varepsilon_{\mathrm{TD}}^{311}=0 \%$ before the laser passes over the gauge volume. Despite the free surfaces close to the left and right edge gauge volumes, the energy input by the laser does still have a noticeable effect, which is indicated by the increase in the strain difference during cooling after the laser passed over the gauge volume. 


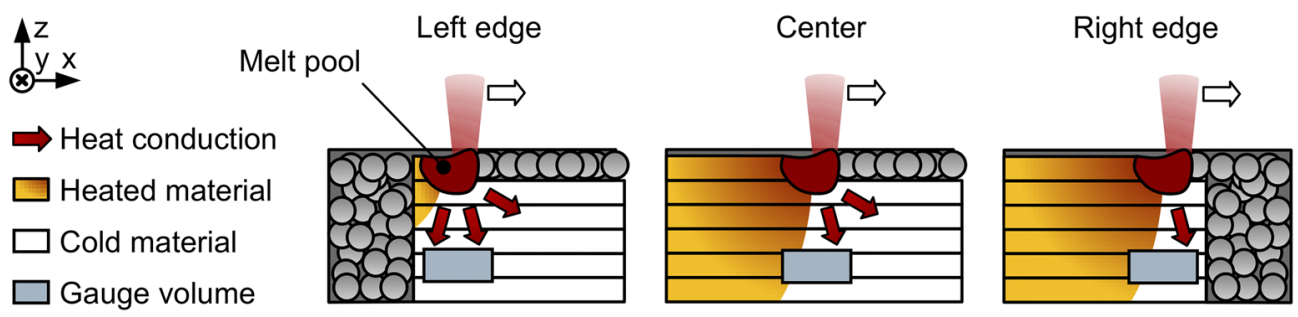

FIG. 7. Schematic diagram of heat conduction from the melt pool and lateral heat accumulation in an LPBF sample.

\section{CONCLUSIONS}

An LPBF system for the realization of in situ $\mathrm{x}$-ray diffraction experiments during the buildup of multi-layer samples was presented. It is based on an industrial system with a custom-built process chamber in order to mimic the industrial process as closely as possible while considering the special requirements to carry out in situ $\mathrm{x}$-ray diffraction experiments. The design of the process chamber allows for observation of arbitrary measuring positions in the sample in the transmission mode while gathering full diffraction rings. First experiments conducted at PETRA III show promising results and give insight into the dynamics of the lattice spacing during the build-up of Inconel 625 samples consisting of 100 layers. Upcoming scientific publications will focus on the results of extensive series of experiments using the here described experimental instrumentation. Further improvements of the instrumentation will address the possibility to gather more temperature information during the process in order to evaluate in-process stresses. The instrumentation is not limited to the single use of Inconel 625 as the powder material but allows for in situ strain analysis during the manufacturing of other commonly used metals and alloys. Furthermore, the examination of texture evolution and phase transformations during the LPBF process are possible future fields of research that can be considered using the presented experimental setup.

\section{ACKNOWLEDGMENTS}

This paper, which is based on the results obtained in project Nos. DFG UH 100/207-1 and DFG RE 688/73-1, was supported by the Deutsche Forschungsgemeinschaft (DFG). Furthermore, we acknowledge Helmholtz-Zentrum Geesthacht (HZG) for the support and the provision of experimental facilities at PETRA III and the provision of peripheral equipment.

\section{DATA AVAILABILITY}

The data that support the findings of this study are available from the corresponding author upon reasonable request.

\section{REFERENCES}

${ }^{1} \mathrm{~T}$. Wohlers, Wohlers Report: 3D Printing and Additive Manufacturing State of the Industry (WOHLERS Associates, Fort Collins, 2018).

${ }^{2}$ M. Seifi, A. Salem, J. Beuth, O. Harrysson, and J. J. Lewandowski, JOM 68, 747 (2016).
${ }^{3}$ E. Uhlmann, G. Gerlitzky, and C. Fleck, in Proceedings of the 28th Annual International Solid Freeform Fabrication Symposium, Austin, Texas (University of Texas, 2017), p. 48.

${ }^{4}$ S. Leuders, M. Thöne, A. Riemer, T. Niendorf, T. Tröster, H. A. Richard, and H. J. Maier, Int. J. Fatigue 48, 300 (2013).

${ }^{5}$ J. L. Bartlett and X. Li, Addit. Manuf. 27, 131 (2019).

${ }^{6}$ L. Parry, I. A. Ashcroft, and R. D. Wildman, Addit. Manuf. 12, 1 (2016).

${ }^{7}$ A. M. Rausch, V. E. Küng, C. Pobel, M. Markl, and C. Körner, Materials 10, 1117 (2017).

${ }^{8}$ T. M. Rodgers, J. D. Madison, and V. Tikare, Comput. Mater. Sci. 135, 78 (2017).

${ }^{9}$ S. K. Everton, M. Hirsch, P. Stravroulakis, R. K. Leach, and A. T. Clare, Mater. Des. 95, 431 (2016).

${ }^{10}$ M. Mani, B. M. Lane, M. A. Donmez, S. C. Feng, and S. P. Moylan, Int. J. Prod. Res. 55, 1400 (2016).

${ }^{11}$ W. Reimers, in Neutrons and Synchrotron Radiation in Engineering Materials Science: From Fundamentals to Material and Component Characterization, edited by W. Reimers, A. R. Pyzalla, A. Schreyer, and H. Clemens (Wiley-VCH, Weinheim, Chichester, 2008), p. 115.

${ }^{12}$ E. Uhlmann, E. Krohmer, F. Hohlstein, and W. Reimers, in Proceedings of the 28th Annual International Solid Freeform Fabrication Symposium, Austin, Texas (University of Texas, 2017), p. 1472.

${ }^{13}$ P. Bidare, I. Bitharas, R. M. Ward, M. M. Attallah, and A. J. Moore, Acta Mater. 142, 107 (2018).

${ }^{14}$ P. Bidare, R. R. J. Maier, R. J. Beck, J. D. Shephard, and A. J. Moore, Addit. Manuf. 16, 177 (2017).

${ }^{15}$ C. Zhao, K. Fezzaa, R. W. Cunningham, H. Wen, F. de Carlo, L. Chen, A. D. Rollett, and T. Sun, Sci, Rep. 7, 3602 (2017).

${ }^{16}$ N. D. Parab, C. Zhao, R. Cunningham, L. I. Escano, K. Fezzaa, W. Everhart, A. D. Rollett, L. Chen, and T. Sun, J. Synchrotron Radiat. 25, 1467 (2018).

${ }^{17}$ Q. Guo, C. Zhao, L. I. Escano, Z. Young, L. Xiong, K. Fezzaa, W. Everhart, B. Brown, T. Sun, and L. Chen, Acta Mater. 151, 169 (2018).

${ }^{18}$ L. I. Escano, N. D. Parab, L. Xiong, Q. Guo, C. Zhao, K. Fezzaa, W. Everhart, T. Sun, and L. Chen, Sci. Rep. 8, 15079 (2018).

${ }^{19}$ L. I. Escano, N. D. Parab, L. Xiong, Q. Guo, C. Zhao, T. Sun, and L. Chen, Synchrotron Radiat. News 32, 9 (2019).

${ }^{20}$ C. Kenel, D. Grolimund, X. Li, E. Panepucci, V. A. Samson, D. F. Sanchez, F. Marone, and C. Leinenbach, Sci. Rep. 7, 16358 (2017).

${ }^{21}$ C. Kenel, D. Grolimund, J. L. Fife, V. A. Samson, S. van Petegem, H. van Swygenhoven, and C. Leinenbach, Scr. Mater. 114, 117 (2016).

${ }^{22}$ C. L. A. Leung, S. Marussi, R. C. Atwood, M. Towrie, P. J. Withers, and P. D. Lee, Nat. Commun. 9, 1355 (2018).

${ }^{23}$ C. L. A. Leung, S. Marussi, M. Towrie, J. del Val Garcia, R. C. Atwood, A. J. Bodey, J. R. Jones, P. J. Withers, and P. D. Lee, Addit. Manuf. 24, 647 (2018).

${ }^{24}$ C. L. A. Leung, S. Marussi, M. Towrie, R. C. Atwood, P. J. Withers, and P. D. Lee, Acta Mater. 166, 294 (2019).

${ }^{25}$ N. P. Calta, J. Wang, A. M. Kiss, A. A. Martin, P. J. Depond, G. M. Guss, V. Thampy, A. Y. Fong, J. N. Weker, K. H. Stone, C. J. Tassone, M. J. Kramer, M. F. Toney, A. van Buuren, and M. J. Matthews, Rev. Sci. Instrum. 89, 055101 (2018). 
${ }^{26}$ A. A. Martin, N. P. Calta, J. A. Hammons, S. A. Khairallah, M. H. Nielsen, R. M. Shuttlesworth, N. Sinclair, M. J. Matthews, J. R. Jeffries, T. M. Willey, and J. R. I. Lee, Mater. Today Adv. 1, 100002 (2019).

${ }^{27}$ A. Bobel, L. G. Hector, I. Chelladurai, A. K. Sachdev, T. Brown, W. A. Poling, R. Kubic, B. Gould, C. Zhao, N. Parab, A. Greco, and T. Sun, Materialia 6, 100306 (2019).

${ }^{28}$ R. Cunningham, C. Zhao, N. Parab, C. Kantzos, J. Pauza, K. Fezzaa, T. Sun, and A. D. Rollett, Science 363, 849 (2019).

${ }^{29}$ A. A. Martin, N. P. Calta, S. A. Khairallah, J. Wang, P. J. Depond, A. Y. Fong, V. Thampy, G. M. Guss, A. M. Kiss, K. H. Stone, C. J. Tassone, J. Nelson Weker, M. F. Toney, T. van Buuren, and M. J. Matthews, Nat. Commun. 10, 1987 (2019).

${ }^{30}$ C. Zhao, Q. Guo, X. Li, N. Parab, K. Fezzaa, W. Tan, L. Chen, and T. Sun, Phys. Rev. X 9, 021052 (2019).

${ }^{31}$ A. M. Kiss, A. Y. Fong, N. P. Calta, V. Thampy, A. A. Martin, P. J. Depond, J. Wang, M. J. Matthews, R. T. Ott, C. J. Tassone, K. H. Stone, M. J. Kramer,
A. van Buuren, M. F. Toney, and J. Nelson Weker, Adv. Eng. Mater. 21, 1900455 (2019).

${ }^{32}$ S. Hocine, H. van Swygenhoven, S. van Petegem, C. S. T. Chang, T. Maimaitiyili, G. Tinti, D. Ferreira Sanchez, D. Grolimund, and N. Casati, Mater. Today 34, 30 (2019).

${ }^{33}$ F. Schmeiser, E. Krohmer, N. Schell, E. Uhlmann, and W. Reimers, Addit. Manuf. 32, 101028 (2020)

${ }^{34}$ N. Schell, A. King, F. Beckmann, T. Fischer, M. Müller, and A. Schreyer, Mater. Sci. Forum 772, 57 (2013)

${ }^{35}$ A. P. Hammersley, S. O. Svensson, M. Hanfland, A. N. Fitch, and D. Hausermann, High Pressure Res. 14, 235 (1996).

${ }^{36}$ M. Newville, T. Stensitzki, D. B. Allen, and A. Ingargiola (2014). "LMFIT: Nonlinear least-square minimization and curve-fitting for Python," Zenodo.

${ }^{37}$ ISO 21432:2005, Non-destructive testing-Standard test method for determining residual stresses by neutron diffraction, Geneva, Switzerland, 2005.

${ }^{38}$ P. Mercelis and J.-P. Kruth, Rapid Prototyping J. 12, 254 (2006). 